\title{
Enhancement of Current Auto- and Cross-Correlations in Double Quantum Dots
}

\author{
G. MichaŁeK And B.R. BuŁKa \\ Institute of Molecular Physics, Polish Academy of Sciences \\ M. Smoluchowskiego 17, 60-179 Poznań, Poland
}

\begin{abstract}
Studies of an enhancement of current shot noises are presented for a device of two large quantum dots coupled capacitively. We analyze current-current correlation functions, and show that a dynamical Coulomb blockade is responsible for an enhancement of the Fano factors. This process can lead also to an electron bunching and positive current cross-correlations. Our theoretical results are discussed in light of recent shot noise experiments.
\end{abstract}

PACS numbers: 73.23.Hk, 73.63.Kv, 73.50.Td

\section{Introduction}

The shot noise analysis gives an additional information about electron-electron correlations, which are present during electronic transport in nanodevices $[1,2]$. In general, the correlations between conducting electrons are negative, which is manifested in a suppression of the shot noise below the Poissonian value $S_{\mathrm{P}}=2 e I$ [2]. In multiterminal devices, current fluctuations can also be studied through cross-correlations between two different branches of the circuit. In such a situation the Fermi statistics of electrons leads to the negative zero-frequency cross-correlations [3]. However, electron-electron interactions can lead in some cases to the positive correlations, e.g. in negative differential resistance (NDR) regions of resonant tunneling diodes [4] or in double quantum dots (QDs) coupled in parallel $[5,6]$. Appearance of the positive cross-correlations in fermionic systems is a characteristic feature of interactions. It has been shown theoretically that the positive cross-correlations can arise in devices with superconducting or ferromagnetic leads, in the Luttinger liquid, in presence of dynamical screening, dynamical channel blockade and strong inelastic scattering (see e.g. $[7,8])$.

Our studies are inspired by recent experiments on the shot noise in two quantum dots capacitively coupled [9]. In this paper, we study an influence of dynamical processes, especially a dynamical Coulomb blockade, on an electron bunching and on an enhancement of auto- and cross-correlations.

\section{Model of double QD and calculations of currents and noises}

The system under consideration is presented in Fig. 1. It is composed of two quantum dots (a top - $t$ and a bottom - b) connected with leads through tunnel junctions and coupled in parallel by a capacitance $C_{\text {int }}$. Each tunnel junction is described by its macroscopic capacitance $C_{\alpha}$ and resistance $R_{\alpha}$. For simplicity we assume that all capacitances of the system are equal to $C_{0}$ (i.e. $C_{\alpha}, C_{\text {int }}=C_{0}$ ), while the resistances $R_{\alpha}$ can be different for each junction $\alpha=\mathrm{Lt}, \mathrm{Rt}, \mathrm{Lb}, \mathrm{Rb}$. We restrict our considerations to the large quantum dots (with a continuous electronic density of states). It is also assumed that the corresponding resistances of the tunnel junctions are much larger than the quantum resistance $R_{\mathrm{Q}}=h / 2 e^{2}$ and an electronic transport is dominated by incoherent, sequential tunneling processes [10]. Tunneling rates through the junctions can be calculated using generalization of the method described e.g. in the paper [11]. For example, for the transfer of one electron from the top QD to the right electrode through the junction Rt one can obtain

$$
\begin{aligned}
\Gamma_{\mathrm{Rt}}^{-} & \left(n_{\mathrm{t}}, n_{\mathrm{b}}\right) \equiv \Gamma_{\mathrm{Rt}}^{-}\left(n_{\mathrm{t}}, n_{\mathrm{b}} ; n_{\mathrm{t}}-1, n_{\mathrm{b}}\right) \\
& =\frac{\Delta F_{\mathrm{Rt}}\left(n_{\mathrm{t}}, n_{\mathrm{b}} ; n_{\mathrm{t}}-1, n_{\mathrm{b}}\right)}{e^{2} R_{\mathrm{Rt}}} \\
& \times\left[1-\exp \left(-\frac{\Delta F_{\mathrm{Rt}}\left(n_{\mathrm{t}}, n_{\mathrm{b}} ; n_{\mathrm{t}}-1, n_{\mathrm{b}}\right)}{k_{\mathrm{B}} T}\right)\right]^{-1},
\end{aligned}
$$

where $e$ denotes charge of an electron and $\Delta F_{\mathrm{Rt}}\left(n_{\mathrm{t}}, n_{\mathrm{b}} ; n_{\mathrm{t}}-1, n_{\mathrm{b}}\right)$ is a difference of free energies of the system for the initial $\left(n_{\mathrm{t}}, n_{\mathrm{b}}\right)$ and the final $\left(n_{\mathrm{t}}-1, n_{\mathrm{b}}\right)$ states, respectively. The free energy of the system consists of potential energies of the electrodes and of electrostatic energies of the charged capacitors [12].

In the stationary state the average current flowing through the junction $\alpha$ can be found from the formula

$$
I_{\alpha}=-e \sum_{n_{\mathrm{t}}, n_{\mathrm{b}}}\left[\Gamma_{\alpha}^{+}\left(n_{\mathrm{t}}, n_{\mathrm{b}}\right)-\Gamma_{\alpha}^{-}\left(n_{\mathrm{t}}, n_{\mathrm{b}}\right)\right] p\left(n_{\mathrm{t}}, n_{\mathrm{t}}\right),
$$

where the steady state probability $p\left(n_{\mathrm{t}}, n_{\mathrm{b}}\right)$ describes the system which contain $n_{\mathrm{t}}$ and $n_{\mathrm{b}}$ additional elec- 


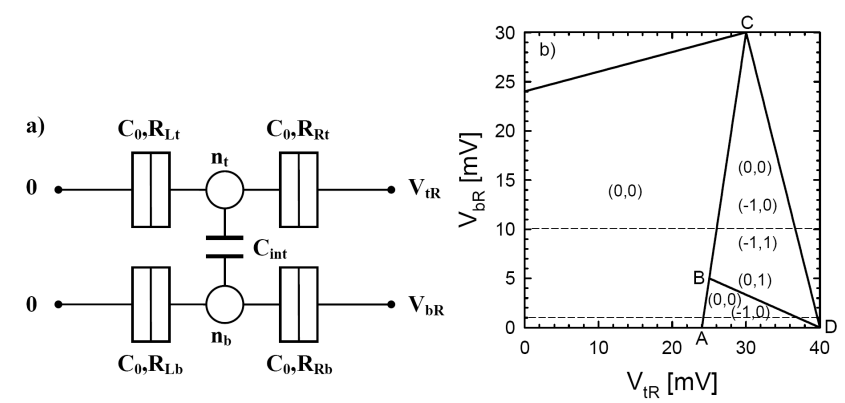

Fig. 1. (a) Schematic view of two quantum dots coupled in parallel. The tunnel junction $\alpha$ is characterized by the capacitance $C_{\alpha}=C_{0}$ and the resistance $R_{\alpha}$, while the dot-dot capacitance is denoted by $C_{\text {int }}$ $\left(C_{\mathrm{int}}=C_{0}\right) . V_{\mathrm{tR}}, V_{\mathrm{bR}}$ are voltages applied to the right electrodes and $n_{\mathrm{t}}, n_{\mathrm{b}}$ denote a number of excess electrons on the top (t) and the bottom (b) QDs, respectively. (b) Stability diagram of available states. Thick lines show threshold voltages $V_{\mathrm{th}}$, where new charge states $\left(n_{\mathrm{t}}, n_{\mathrm{b}}\right)$ are activated. The calculations have been performed for $C_{0}=2 \mathrm{aF}$ and $T=0.011 \mathrm{~K}$.

trons on the top and the bottom QDs, respectively. The probability can be found from the master equation $\partial \hat{p}\left(n_{\mathrm{t}}, n_{\mathrm{b}} ; t\right) / \partial t=\hat{M} \hat{p}\left(n_{1}, n_{2} ; t\right)$ with the left hand side equal to zero. The tunnel matrix $\hat{M}$ depends on the tunneling rates $\Gamma_{\alpha}^{ \pm}\left(n_{\mathrm{t}}, n_{\mathrm{b}}\right)$.

To analyze fluctuations in the system we extend the generation-recombination approach [13] for multi-electron channels by a generalization of the method developed for spinless electrons in a single-electron transistor [14]. According to this procedure the Fourier transform of the correlation function of a quantity $X$ is

$$
\begin{aligned}
& S_{X X}(\omega)=4 \sum_{n_{\mathrm{t}}, n_{\mathrm{b}} ; n_{\mathrm{t}}^{\prime}, n_{\mathrm{b}}^{\prime}} X\left(n_{\mathrm{t}}, n_{\mathrm{b}}\right) G_{n_{\mathrm{t}}, n_{\mathrm{b}} ; n_{\mathrm{t}}^{\prime}, n_{\mathrm{b}}^{\prime}}(\omega) \\
& \quad \times X\left(n_{\mathrm{t}}^{\prime}, n_{\mathrm{b}}^{\prime}\right) p\left(n_{\mathrm{t}}^{\prime}, n_{\mathrm{b}}^{\prime}\right),
\end{aligned}
$$

where $\hat{G}(\omega) \equiv 1 /(\mathrm{i} \omega \hat{1}-\hat{M})-\hat{p} / \mathrm{i} \omega$. The auto- and the cross-correlation functions between the currents $I_{\alpha}$ and $I_{\beta}$ can be expressed by the formula

$$
S_{\alpha, \beta}(\omega)=\delta_{\alpha \beta} S_{\alpha}^{\mathrm{Sch}}+S_{\alpha, \beta}^{\mathrm{c}}(\omega),
$$

where $S_{\alpha}^{\text {Sch }}$ is the high frequency limit $(\omega \rightarrow \infty)$ of the shot noise (the Schottky noise), and the second part $S_{\alpha, \beta}^{\mathrm{c}}$ is frequency dependent [14].

\section{Enhancement of current shot noise}

We have numerically calculated currents and their fluctuations as a function of bias voltages applied to the top $\left(V_{\mathrm{tR}}\right)$ and the bottom $\left(V_{\mathrm{bR}}\right)$ QDs. Figure $1 \mathrm{~b}$ shows the stability diagram of available states. For small voltages the system is in the state $(0,0)$ and the current cannot flow because of the Coulomb blockade. With increasing voltages new charge states $\left(n_{\mathrm{t}}, n_{\mathrm{b}}\right)$ become available for tunneling events. The thick lines in Fig. 1b denote the threshold voltages $V_{\mathrm{th}}$ between initial $\left(n_{\mathrm{t}}, n_{\mathrm{b}}\right)$ and final
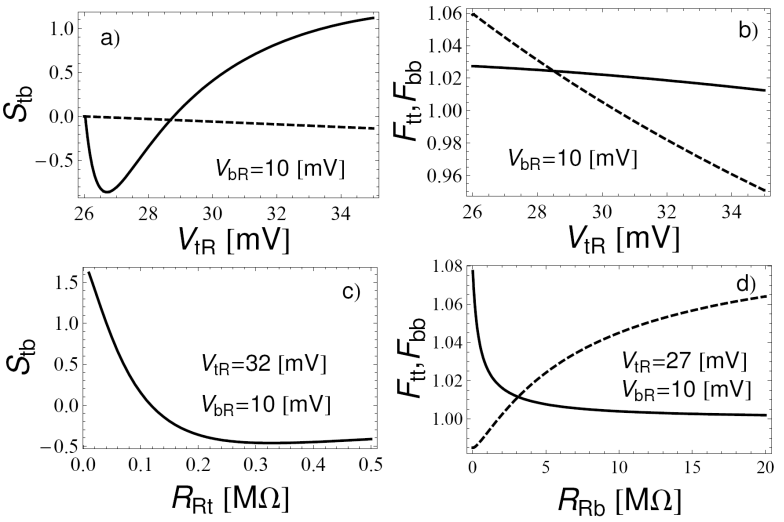

Fig. 2. (a) Cross-correlation function $S_{\mathrm{tb}} \equiv$ $S_{\mathrm{tR}, \mathrm{bR}}(\omega=0)$ versus $V_{\mathrm{tR}}$ for $R_{\mathrm{Lt}}=1 \mathrm{M} \Omega$, $R_{\mathrm{Lb}}=R_{\mathrm{Rb}}=2 \mathrm{M} \Omega$. Solid line: $R_{\mathrm{Rt}}=0.05 \mathrm{M} \Omega$; dashed line: $R_{\mathrm{Rt}}=3 \mathrm{M} \Omega$. (b) The Fano factors $F_{\mathrm{tt}} \equiv$ $S_{\mathrm{tR}, \mathrm{tR}}(\omega=0) / 2 e I_{\mathrm{tR}}$ and $F_{\mathrm{bb}} \equiv S_{\mathrm{bR}, \mathrm{bR}}(\omega=0) / 2 e I_{\mathrm{bR}}$ versus $V_{\mathrm{tR}}$ for $R_{\mathrm{Lt}}=1 \mathrm{M} \Omega, R_{\mathrm{Rt}}=3 \mathrm{M} \Omega, R_{\mathrm{Lb}}=2 \mathrm{M} \Omega$. Solid line: $F_{\mathrm{bb}}$ for $R_{\mathrm{Rb}}=1 \mathrm{M} \Omega$; dashed line: $F_{\mathrm{tt}}$ for $R_{\mathrm{Rb}}=10 \mathrm{M} \Omega$. (c) Cross-correlation $S_{\mathrm{tb}}$ as a function of resistance $R_{\mathrm{Rt}}$ for $V_{\mathrm{tR}}=32 \mathrm{mV}$. (d) Fano factors $F_{\mathrm{bb}}$ (solid line) and $F_{\mathrm{tt}}$ (dashed line) as a function of resistance $R_{\mathrm{Rb}}$ for $V_{\mathrm{tR}}=27 \mathrm{mV}$. The other parameters are: $C_{0}=2 \mathrm{aF}, V_{\mathrm{bR}}=10 \mathrm{mV}, T=0.011 \mathrm{~K}$.

$\left(n_{\mathrm{t}}^{\prime}, n_{\mathrm{b}}^{\prime}\right)$ states. One can easily find $V_{\mathrm{th}}$ solving the equation $\Delta F_{\alpha}\left(n_{\mathrm{t}}, n_{\mathrm{b}} ; n_{\mathrm{t}}^{\prime}, n_{\mathrm{b}}^{\prime}\right)=0$. For example, in the triangle ABD the additional state $(-1,0)$ is opened for the tunneling processes and a current can flow through the top QD, see Fig. $1 \mathrm{~b}$. For the set of voltages $V_{\mathrm{tR}}$ and $V_{\mathrm{bR}}$ corresponding to the large triangle BCD, four charge states $(0,0),(-1,0),(-1,1),(0,1)$ are activated, so currents can flow through the both QDs.

The cross-correlation $S_{\text {tb }}$ (Fig. 2a) shows a positive and a negative current correlations. In a case of a large resistance of the output junction $\left(R_{\mathrm{Rt}}=3 \mathrm{M} \Omega\right.$ and larger $)$ the cross-correlations are negative, as one can expect for free electrons. However, for a smaller output resistance $\left(R_{\mathrm{Rt}}=0.05 \mathrm{M} \Omega\right) S_{\mathrm{tb}}$ (solid curve) is negative at small $V_{\mathrm{tR}}$ and becomes positive for larger voltages (see Fig. 2c). In this case more electrons leave the top QD through the right tunnel junction than arrive from the left electrode. Hence, the corresponding charge accumulation on the top QD is negative. This charge accumulation monotonically decreases with increasing $V_{\mathrm{tR}}$. The large negative charge accumulation on the top dot prefers the positive charge accumulation on the bottom dot. The charges accumulated on the both dots strongly interact because the dotdot coupling $C_{\text {int }}$ is strong. Charge fluctuations at the top QD lead to negative cross-correlations. On the other hand, fluctuations at the bottom QD are strongly activated for larger voltages. These fluctuations are dominating and it results in positive cross-correlations. A similar effect, the gate-controlled sign reversal of noise cross-correlations, has been found in experiments with double QDs capacitively coupled in parallel in the Coulomb blockade regime [9]. 
The calculations of the auto-correlation $F_{\mathrm{tt}}$ shows that for small voltages (e.g. $V_{\mathrm{bR}}=1 \mathrm{mV}$ ) applied to the bottom QD the Fano factor decreases below the Poissonian value due to the negative correlations between tunneling events in the same branch of the circuit. In such a case the system can be treated as the double tunnel junction because the current can flow only through the top QD. However, for higher voltages (e.g. $V_{\mathrm{bR}}=10 \mathrm{mV}$ ) the super Poissonian current noise can appear (Fig. 2b) for the large resistance of the output junction $\left(R_{\mathrm{Rt}}=3 \mathrm{M} \Omega\right.$ and larger). A strong dot-dot interaction, which leads to a competition between charge channels for the top and the bottom QDs, is responsible for the enhancement of the current noise. As a result, a dynamical Coulomb blockade appears which leads to bunching of electrons and to the super Poissonian current noise. The increasing voltage $V_{\mathrm{tR}}$ influences the dynamical Coulomb blockade leading to reduction of the shot noise. For high voltages $V_{\mathrm{tR}}$ bunching vanishes for the top QD, so the Fano factor $F_{\mathrm{tt}}$ decreases below the Poissonian value.

The influence of the output resistances $R_{\mathrm{Rt}}$ and $R_{\mathrm{Rb}}$ on the positive cross-correlations and the enhancement of the Fano factors is shown in Fig. 2c,d. We have found that an increase in the output resistance $R_{\mathrm{Rt}}$ leads to the large reduction of the charge fluctuations, so the positive cross-correlations very quickly vanish, see Fig. 2c. The super-Poissonian Fano factor $F_{\mathrm{tt}}$ seen for the large resistances $R_{\mathrm{Rb}}$ is due to an enhancement of the outgoing tunneling processes $\Gamma_{\mathrm{Rt}}^{-}(0,0)$ and $\Gamma_{\mathrm{Rt}}^{-}(0,1)$. The Fano factor $F_{\mathrm{bb}}$ decreases with increasing resistance $R_{\mathrm{Rb}}$, but always $F_{\mathrm{bb}}>1$, see Fig. 2 d. An enhancement of the Fano factor $F_{\mathrm{bb}}$ for small resistances $R_{\mathrm{Rb}}$ is due to the large activation of the outgoing processes $\Gamma_{\mathrm{Rb}}^{-}(0,1)$ and $\Gamma_{\mathrm{Rb}}^{-}(-1,1)$.

To summarize, we have found the dynamical Coulomb blockade due to the competition between tunneling rates for the top and the bottom QDs. This leads to the electron bunching which is responsible for the positive cross- -correlations and an enhancement of the auto-correlations in double QDs systems with asymmetrical resistances.

\section{Acknowledgments}

The work was supported as part of the European Science Foundation EUROCORES Programme FoNE by funds from the Ministry of Science and Higher Education and EC 6FP (contract No. ERAS-CT-2003-980409).

\section{References}

[1] Sh. Kogan, Electronic Noise and Fluctuations in Solids, Cambridge University Press, Cambridge 1996.

[2] Ya.M. Blanter, M. Büttiker, Phys. Rep. 336, 1 (2000).

[3] M. Büttiker, Phys. Rev. B 46, 12485 (1992).

[4] G. Iannaccone, G. Lombardi, M. Macucci, B. Pellegrini, Phys. Rev. Lett. 80, 1054 (1998).

[5] S. Haupt, J. Aghassi, M.H. Hettler, G. Schön, arXiv:0802.3579v1.

[6] G. Michałek, B.R. Bułka, Eur. Phys. J. B 28, 121 (2002).

[7] A. Cottet, W. Belzig, C. Bruder, Phys. Rev. B 70, 115315 (2004).

[8] A. Cottet, W. Belzig, C. Bruder, Phys. Rev. Lett. 92, 206801 (2004).

[9] D.T. McClure, L. DiCarlo, Y. Zhang, H.-A. Engel, C.M. Marcus, M.P. Hanson, A.C. Gossard, Phys. Rev. Lett. 98, 056801 (2007).

[10] G. Schön, Quantum Transport and Dissipation, Wiley-VCH Verlag, New York 1998, p. 149.

[11] M. Amman, R. Wilkins, E. Ben-Jacob, P.D. Maker, R.C. Jaklevic, Phys. Rev. B 43, 1146 (1991).

[12] J.A. Melsen, U. Hanke, H.-O. Müller, K.-A. Chao, Phys. Rev. B 55, 10638 (1997).

[13] K.M. van Vliet, J.R. Fassett, Fluctuation Phenomena in Solids, Academic Press, New York 1965, p. 267.

[14] A.N. Korotkov, Phys. Rev. B 49, 10381 (1994). 